\title{
UTILISATION DE LA DELTAMÉTHRINE CONTRE \\ MATSUCOCCUS FEYTAUDI DUC., LA COCHENILLE DU PIN MARITIME
}

\author{
P.R. CARLE - H. DELAGES
}

Le dépérissement du Pin maritime en forêt méditerranéenne fait l'objet d'une abondante littérature (Joly 1963, Blanck 1966, Schvester 1967, Carle 1968, 1973). Très vite, au cours de cette étude et notamment dès la mise en évidence du rôle de ravageur primaire de la Cochenille Matsucoccus feytaudi (Duc.), les recherches s'orientent d'une part vers des méthodes sylvicoles, d'autre part vers une lutte chimique. Plusieurs solutions se révèlent intéressantes, telle celle de la destruction des larves après éclosion (Schvester 1967, Blanck et Gayraud 1969) ou celle des formes adultes (Carle et al. 1970), de sorte qu'un traitement curatif peut être conseillé entre début février et la mi mai. Dès lors et à raison d'une application insecticide annuelle, biennale voire même triennale, il devient possible d'assurer une bonne élimination des populations du ravageur primaire, la destruction des insectes secondaires pionniers comme Pissodes notatus (L) et la disparition des symptômes de dépérissement sur houppier ("flagging ") et sur tronc (exsudations) (Carle et al. 1970). C'est sur de tels critères et après plusieurs séries d'essais qu'ont été finalement sélectionnés le diéthion et l'oléoparathion aux doses respectives de 100 et $25 \mathrm{~g}$ matière active/ $\mathrm{hl}$ en pulvérisation classique et avec fort mouillage des fûts (Carle 1983). Depuis 1972, cette méthode de lutte est appliquée chaque année contre la Cochenille sur les Pins maritimes utilisés comme arbres d'alignement et d'ornement ou constituant des espaces verts, bosquets et petits boisements littoraux (Delages 1978). Le bilan actuel d'une telle lutte, jusqu'ici confinée en France à quelques centaines de milliers d'arbres, demeure néanmoins très positif dans la mesure où l'intervention chimique reste encore aujourd'hui le seul recours possible.

En 1984, et conformément aux prévisions, l'aire géographique de la Cochenille s'étend à l'Espagne (Cadahia et Montoya 1967) et à l'Italie, avec des risques d'extension sur plus de 160000 hectares de Pins maritimes. Déjà dans les peuplements littoraux de Ligurie, des symptômes de dépérissement apparaissent. En outre, dans plusieurs pays (U.S.A., Pologne, etc), d'autres espèces de Cochenilles, notamment du genre Matsucoccus (Mc Kenzie et al. 1948, Bean et Godwin 1955, Duda 1961) posent de graves problèmes forestiers restés jusqu'ici sans solution. De ce fait, la méthode de lutte mise au point contre Matsucoccus feytaudi en région méridionale française peut offrir des perspectives d'application dans d'autres contextes forestiers. Toutefois, son principal inconvénient réside dans l'usage de pesticides qui, comme le diéthion, possèdent une toxicité relative élevée (cœfficient de sécurité pratique $=21$ ) et une 
persistance d'action relativement réduite. Une solution consistait donc à rechercher parmi les nouveaux produits phytosanitaires disponibles celui ou ceux qui, assurant les mêmes avantages que le diéthion, n'en présenteraient pas les inconvénients. Dans ce but, nous avons choisi d'effectuer des essais avec la deltaméthrine, insecticide pyréthrinoïde photostable peu toxique (cœfficient de sécurité pratique $=1350$ ) à forte rémanence et d'activité biologique encore inégalée, maintenant largement utilisé en milieux domestique, agricole et vétérinaire (in : Roussel Uclaf 1982) et recommandé en milieu forestier (Carle et Fourcaud, Monographie deltaméthrine, tome 2, à paraître).

\section{DISPOSITIF D'EXPÉRIMENTATION}

Les essais ont été réalisés dans un peuplement de Pins maritimes de bord de route à couvert nettoyé, âgés de 15 à 25 ans, visiblement issus de régénération naturelle après incendie, et situés sur la route départementale $n^{\circ} 92$ au lieu-dit "Le Grand Duc" sur la commune de Mandelieu (Alpes-Maritimes). Ce peuplement occupe le versant nord d'une crête orientée O-E et bénéficie d'un ensoleillement maximum à une altitude de 400 mètres. Douze placettes réparties en 3 blocs de 4 traitements ( $A, B, C, T$ ) ont été ménagées comprenant chacune 15 pins de tailles différentes, comprises entre 3 et $7 \mathrm{~m}$ de hauteur, de diamètres, de ports sensiblement variables et infestés par Matsucoccus. Chaque placette a été délimitée et chaque Pin a été numéroté et repéré sur plan.

Le produit étudié est la deltaméthrine en formulation K-Othrine CE 25 (concentré émulsionnable à $25 \mathrm{~g}$ matière active de deltaméthrine par litre). Trois doses ont été concurremment expérimentées, avec trois répétitions pour chaque: $1,25 \mathrm{~g} / \mathrm{hl}$ (traitement A), $2,5 \mathrm{~g}$ (traitement B) et $5 \mathrm{~g}$

Tableau 1

Répartition avant traitement des captures de jeunes larves de Matsucoccus feytaudi à $\mathrm{J}-1$ selon les blocs

\begin{tabular}{|c|c|c|c|c|c|c|c|c|c|c|c|c|}
\hline Traitement $\ldots \ldots \ldots \ldots \ldots \ldots \ldots \ldots$ & \multicolumn{3}{|c|}{$\mathrm{T}$} & \multicolumn{3}{|c|}{ A } & \multicolumn{3}{|c|}{ B } & \multicolumn{3}{|c|}{ C } \\
\hline 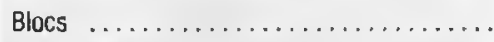 & 1 & 2 & 3 & 1 & 2 & 3 & 1 & 2 & 3 & 1 & 2 & 3 \\
\hline $\begin{array}{l}\text { Moyenne des captures de larves par centi- } \\
\text { mètre carré de bande piège/arbre ........ }\end{array}$ & 169 & 653 & 1498 & 760 & 299 & 124 & 67 & 74 & 668 & 646 & 299 & 34 \\
\hline Total & \multicolumn{3}{|c|}{2320} & \multicolumn{3}{|c|}{1183} & \multicolumn{3}{|c|}{809} & \multicolumn{3}{|c|}{979} \\
\hline
\end{tabular}

Tableau II

Résultats des essais deltaméthrine sur larves néonées de Matsucoccus teytaudi

\begin{tabular}{|c|c|r|r|r|r|r|r|}
\hline \multirow{2}{*}{$\begin{array}{c}\text { Date des } \\
\text { observations }\end{array}$} & Témoin T & \multicolumn{2}{|c|}{$\begin{array}{c}\text { Traitement A } \\
1,25 \mathrm{~g} / \mathrm{hl}\end{array}$} & \multicolumn{2}{|c|}{$\begin{array}{c}\text { Traitement B } \\
2,5 \mathrm{~g} / \mathrm{hl}\end{array}$} & \multicolumn{2}{|c|}{$\begin{array}{c}\text { Traitement C } \\
5 \mathrm{~g} / \mathrm{hl}\end{array}$} \\
\cline { 2 - 8 } & 1 & 1 & 2 & 1 & 2 & 1 & 2 \\
\hline $\mathrm{J}-1$ & 2320 & 1182 & 0 & 808 & 0 & 979 & 0 \\
$\mathrm{~J}+4$ & 2238 & 175 & 84,7 & 50 & 93,6 & 25 & 97,4 \\
$\mathrm{~J}+7$ & 4695 & 158 & 93,4 & 39 & 97,6 & 25 & 98,7 \\
$\mathrm{~J}+14$ & 5830 & 1140 & 61,6 & 322 & 84,1 & 136 & 94,5 \\
$\mathrm{~J}+22$ & 426 & 496 & 0 & 59 & 59,4 & 30 & 83,9 \\
$\mathrm{~J}+30$ & 257 & 123 & 6,1 & 55 & 38,9 & 4 & 96,3 \\
\hline
\end{tabular}

(1) Moyenne des captures par $\mathrm{cm}^{2}$ de bande piege.

(2) Taux d'efficacitè relative par rapport au tèmoin selon la date de capture (en \%). 
(traitement $\mathrm{C}$ ) et 3 parcelles témoin (traitement $\mathrm{T}$ ). Le matériel de traitement utilisé était un camion autopompe à pression de $15 \mathrm{~kg} / \mathrm{cm}^{2}$ et la consommation moyenne de bouillie a été de l'ordre de 60 litres de solution pour 15 arbres par placette.

Le traitement a été réalisé le 24 avril 1984, dans de bonnes conditions et par beau temps clair ; i) a été suivi d'une période de séchage tout à fait satisfaisante.

Les contrôles d'efficacité des traitements ont été effectués sur 10 arbres par parcelle, les 5 autres étant mis en réserve. La technique de contrôle, déjà utilisée dans les précédents essais contre Matsucoccus (Carle et al. 1970), repose sur le piégeage périodique des larves néonées sur des bandes à face adhésive disposée à l'extérieur qui sont posées de manière à encercler le fût de l'arbre, traité ou témoin, au niveau où l'écorce commence à présenter des fissurations peu profondes. La durée de maintien des pièges est de 24 heures ou 36 heures à $\mathrm{J}+22$. Le dénombrement des populations de ces jeunes larves engluées sur la bande est réalisé en laboratoire sous microscope stéréoscopique par calcul de la densité moyenne d'infestation par $\mathrm{cm}^{2}$ de bande lue à l'aide d'un appareillage approprié et selon des modalités parfaitement codifiées.

Des contrôles de population effectués selon cette technique sont opérés sur chacun des 10 arbres pour les 12 placettes choisies la veille du jour $\mathrm{J}$ du traitement puis à $\mathrm{J}+4, \mathrm{~J}+7, \mathrm{~J}+14$, $J+22$ et $J+30$.

\section{RÉSULTATS}

Les contrôles initiaux de population réalisés à $\mathrm{J}-1$ (tableau I) montrent, d'une part une plus grande abondance des captures dans les parcelles du bloc témoin, ce qui est l'indice de populations plus élevées, et d'autre part une équivalence de captures dans les parcelles des blocs devant être soumis aux traitements, toutes conditions particulièrement favorables à l'étude entreprise.

En raison de la difficulté d'évaluation de l'activité insecticide directe sur les jeunes larves par suite d'une impossibilité de dénombrement des individus morts, nous procédons à une mesure indirecte par estimation quantitative des captures sur bandes pièges et analyse comparative des résultats.

Une analyse critique de ces résultats permet de constater un effet dose de l'insecticide, aussi bien sur l'efficacité immédiate à $J+4$ que sur l'activité résiduelle à $J+30$ avec une nette régression d'intensité tout au long de la période d'efficacité. A la plus forte dose, cette activité résiduelle se maintient à un niveau très élevé (95\% d'efficacité) même au bout de 15 jours (tableau II, J + 14).

En outre, le taux d'efficacité relative des traitements, calculé par rapport à l'évolution des populations dans le bloc témoin (tableau II) fait apparaître l'excellente efficacité de la aeltaméthrine durant toute la période de sortie des larves néonées. Cette efficacité est due pour l'essentiel à la très grande activité du produit et à sa persistance sur les fûts traités, persistance qui, à la dose la plus élevée $(5 \mathrm{~g})$, est toujours perceptible même 22 jours après traitement. Au delà, l'effet résiduel du produit est moins bien perçu car il ne s'exerce en fait que sur une infime partie de la population totale $(4,3 \%)$.

Ramenée à l'ensemble de la population, l'efficacité de ces traitements peut être évaluée par cumul des efficacités observées dans chacun des contrôles en excluant les populations relevées à $\mathrm{J}-1$ (figure 1). Ainsi, la dose de $5 \mathrm{~g} / \mathrm{hl}$ assure une excellente protection avec une activité globale de $96,1 \%$ et une persistance de plus de 30 jours, soit durant une période suffisamment 


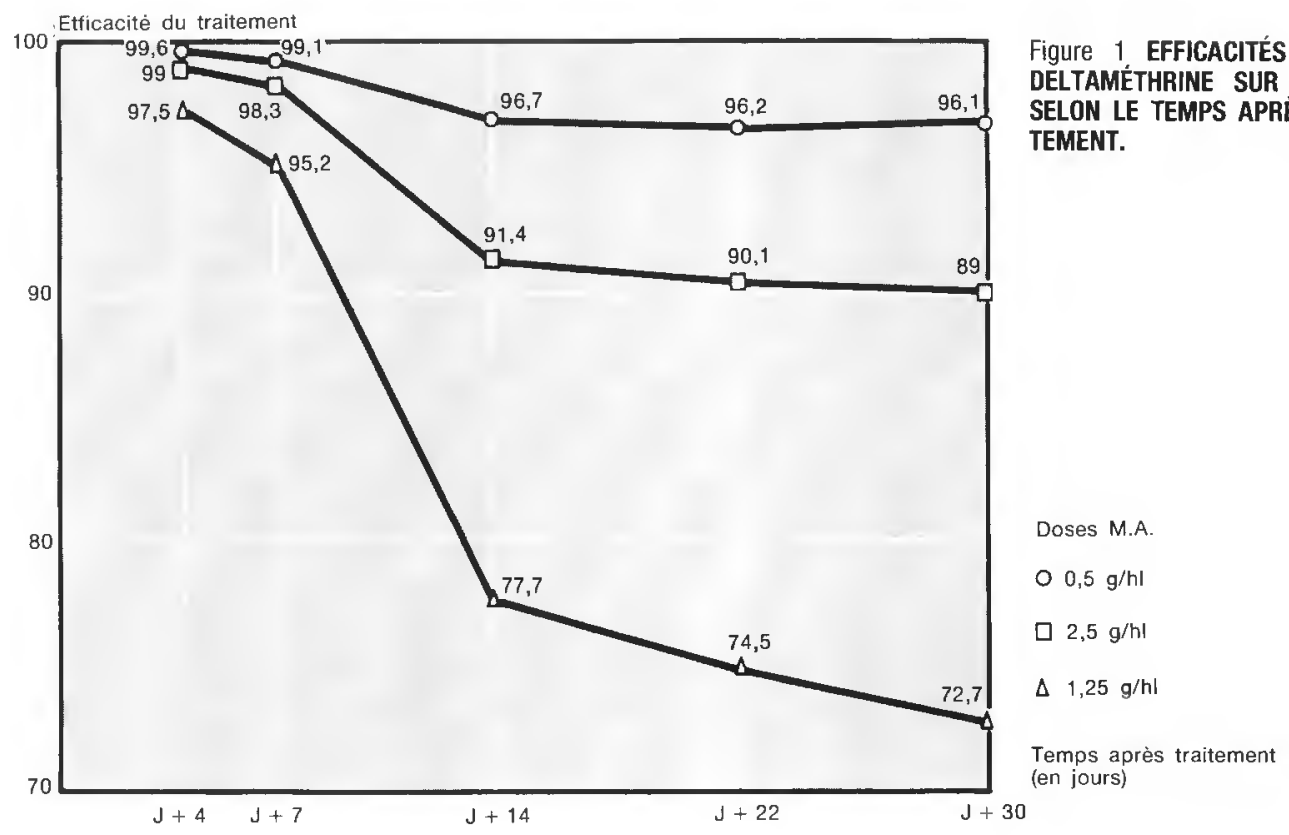

longue pour couvrir toute la phase de sortie de la population larvaire. Comparativement, la dose de $2,5 \mathrm{~g} / \mathrm{hl}$ donne des résultats légèrement inférieurs mais cependant acceptables avec une activité globale de $89 \%$ après 30 jours. Enfin, la dose de $1,25 \mathrm{~g} / \mathrm{hl}$ avec $72,7 \%$ d'efficacité globale apparaît nettement insuffisante.

\section{CONCLUSIONS}

Les essais réalisés en avril 1984, sur les larves néonées de la Cochenille Matsucoccus feytaudi démontrent l'excellente activité de la deltaméthrine à la dose de $5 \mathrm{~g} \mathrm{MA} / \mathrm{hl}$ et une activité encore acceptable à $2,5 \mathrm{~g} / \mathrm{hl}$. Des doses inférieures ne paraissent pas assurer une persistance d'action suffisamment longue de l'insecticide pour couvrir la majeure partie de la période d'éclosion des larves. II n'est cependant pas exclu que des formulations différentes du concentré émulsionnable (CE) employé dans ces essais et telles que des formulations huileuses pour traitements à bas volume ou ultra bas volume ou des microcapsules, etc... ne puissent donner satisfaction à des doses plus réduites.

Comparativement aux résultats précédemment enregistrés avec le diéthion, et même si, pour des raisons techniques, ce pesticide n'a pu être introduit dans nos essais, il apparaît que l'activité biologique démontrée ici par la deltaméthrine est du même ordre que celle déjà obtenue avec ce produit. De ce fait, la faible dose d'utilisation du pyréthrinoïde et sa toxicité intrinsèque réduite sont des arguments économiques et environnementaux particulièrement favorables à son utilisation sur larves de Matsucoccus. En outre, il convient de signaler sa possibilité d'emploi sur les formes adultes de la Cochenille durant leur période d'émergence en février-mars. D'ailleurs, en raison de sa thermodépendance négative (Carle 1982), la deltaméthrine appliquée plus précocement en saison, donc sous des températures plus basses, devrait présenter un effet insecticide ascru et une persistance d'action encore améliorée tout en respectant la faune auxiliaire inactive à cette époque de l'année. 
Enfin, en ce qui concerne les modalités d'application des traitements, nous avons pu remarquer que des interventions chimiques régulières et efficaces contre Matsucoccus provoquaient à la longue une réaction morphologique des écorces des pins aboutissant à une quasi fermeture de leur clivage. Ce phénomène induit nécessairement une modification importante dans la répartition des populations de Matsucoccus sur l'arbre et provoque par contrecoup la colonisation préférentielle du houppier. De ce fait, et pour une meilleure efficacité des traitements contre cette Cochenille, il nous paraît opportun de proposer que les applications insecticides préférentiellement recommandées sur fût soient également dirigées aussi bien sur fût que sur houppier. En outre, bien que jusqu'ici le ruissellement ait été intentionnellement recherché avec le diéthion, une telle opération paraît superflue avec la deltaméthrine, compte tenu de son excellente adhésivité sur les substrats végétaux de nature corticale.
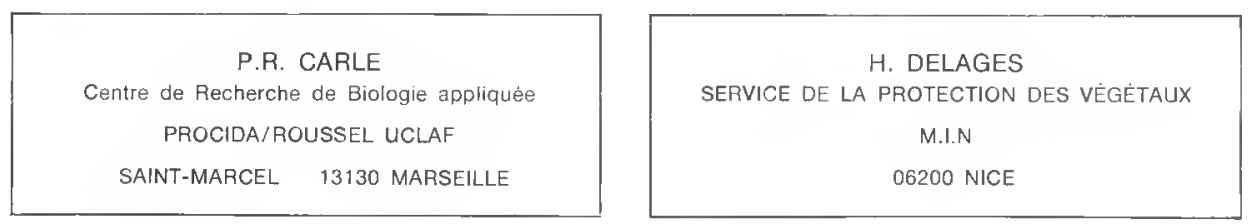

\section{BIBLIOGRAPHIE}

BEAN (J.L.), GODWIN (P.A.). - Description and bionomics of a new red pine scale (Matsucoccus resinosae Hemip. Coccoidae on Pinus resinosa). - Forest Science, vol. 1, $n^{\circ} 2,1955$, pp. 174-176.

BLANCK (A.). - Le rôle de la Cochenille Matsucoccus feytaudi (Homopt. Coccoidae) dans le dépérissement du Pin maritime de la forêt des Maures. - Phytoma, $\mathrm{n}^{\circ}$ 175, 1966, pp. 15-26.

BLANCK (A.), GAYRAUD (Y.). - Le dépérissement du Pin maritime dans le Sud-Est de la France par l'action de la Cochenille Matsucoccus feytaudi. - Phytoma, n ${ }^{\circ} 207,1969$, pp. 2-12.

CADAHIA (D.), MONTOYA (R.). - Prospeccion de Matsucoccus feytaudi Duc y Matsucoccus pini Green (Homoptera Margarodidae) en la mitad Norte de Espana . - Bol. Serv. Plag. For., $n^{\circ} 22,1968$, pp. 133-134.

CARLE (P.R.). - Le dépérissement du Pin maritime dans le Var. Epidémiologie - Symptomatologie - Cause primaire. - Revue forestière française, $\mathrm{n}^{\circ} 3,1968$, pp. 185-203.

CARLE (P.R.). - Le dépérissement du Pin mesogéen en Provence. - Thèse, 1973. - $174 \mathrm{p}$.

CARLE (P.R.). - Destruction des insectes domestiques. - In : Monographie Deltaméthrine. - Roussel Uclaf Ed., 1982. - pp. 233-259.

CARLE (P.R.), FOURCAUD (A.). - Utilisation de la deltaméthrine contre les insectes forestiers. - à paraître.

CARLE (P.R.), RIOM (J.), SCHVESTER (D.). - Le dépérissement du Pin maritime dans le Var. Etat actuel du probleme Matsucoccus feytaudi. - Revue forestière française, numéro spécial "Lutte biologique en forêt ", 1970 , pp. $240-246$.

DELAGES (H.). - Lutte contre la Cochenille des Pins maritimes. - Phytoma, n 297,1978, pp. $19-23$.

DUDA (E.J.). - Some aspects of the biology and ecology of the red pine scale Matsucoccus resinosae $B$. et B. - Doctoral dissertation. - Syracuse (U.S.A.): University of Massachussetts, 1961.

JOLY (R.). - Matsucoccus feytaudi Duc. Nouvelles stations en France. Son importance forestière. - Revue forestière française, $\mathrm{n}^{\circ} 3,1963$, pp. 203-207.

MC KENZIE (H.L.), GILL (L.S.), ELLIS (D.E.). - The prescott scale (Matsucoccus vexillo), an association organisms that cause flagging injury to ponderosa pine in the Southwest. - J. Agric. Research, vol, 76 , $n^{\circ} 2,1948$, pp. 33-52.

ROUSSEL UCLAF. - Monographie Deltaméthrine. - Roussel Uclaf Ed., 1982. - $412 \mathrm{p}$.

SCHVESTER (D.). - Observations générales sur le dépérissement du Pin maritime dans les Maures. - Revue forestière française, $\mathrm{n}^{\circ} 6,1967, \mathrm{pp} .373-385$. 